\title{
Topological excitation in the Fractional Quantum Hall system
}

\author{
Yi-shi Duan ${ }^{1}$, Peng-ming Zhang ${ }^{1 *}$ and Hong Zhang ${ }^{2}$ \\ ${ }^{1}$ Institute of Theoretical Physics, Lanzhou University-Lanzhou, 730000, PRC \\ ${ }^{2}$ Department of Physics and Centre for Nonlinear Studies Hong Kong Baptist \\ University-Hong Kong, PRC
}

(October 24, 2018)

\begin{abstract}
Two kinds of topological excitations, vortices and skyrmions, are studied in the frame of Ginzburg-Landau theory. We obtain the rigorous relation between the topological excitation and the order parameters in the fractional quantum Hall systems. We also discuss the evolution of the vortices in fractional quantum Hall systems.
\end{abstract}

PACS numbers: 73.40.Hm; 02.40.-k; 12.93.Dc

Typeset using REVTEX

*Anthor to whom correspondence should be addressed. Email: zhpm@lzu.edu.cn 


\section{INTRODUCTION}

The fractional quantum Hall (FQH) effect appears in two-dimensional (2D) election systems in a strong magnetic field. Since its discovery in 1982 [1,2], experiments on FQH systems have continued to reveal many new phenomena and surprises. These, together with the observed rich hierarchical structures [3], indicate that electron systems that demonstrate a FQH effect (those systems are called FQH liquids) contain extremely rich internal structures. There are two kinds of topological excitations in single-layer quantum Hall systems. When the system is fully polarized, the relevant charged quasiparticles are topological vortices which carry the $U(1)$ topological charges. But for a weak Zeeman coupling, it has been suggested that [0] the lowest energy quasiparticles at filling factor $\nu=1 / m$ are skyrmions or slowly varying spin textures which carry the $S U(2)$ topological charges.

In this paper, we consider the topological properties of vortices and skyrmions in terms of the $\phi$-mapping topological current theory [6,7]. The inner structure of the vortex threecurrent is given with the spinless wave function in the fully polarized system. Furthermore, the evolution of the vortex is also investigated. And one sees that the vortices generate or annihilate at the limit points and encounter, split, or merge at the bifurcation points of the spinless wave function. Based on the gauge potential decomposition theory [13] the skyrmion three-current in the depolarized system is investigated from a kind of new viewpoint.

\section{VORTEX EXCITATION}

In this section we discuss the vortex excitation of a FQH system in the frame of GL theory. It is well-known that the topological vortices exist in the fully polarized system, the spinless wave function can be denoted as

$$
\psi(\vec{r}, t)=\phi^{1}(\vec{r}, t)+i \phi^{2}(\vec{r}, t)=|\phi| n(\vec{r}, t)
$$

where $n(\vec{r}, t)=n^{1}(\vec{r}, t)+i n^{2}(\vec{r}, t)$ and $n^{a}(a=1,2)$ is the two-dimensional unit vector field

$$
n^{a}(\vec{r}, t)=\phi^{a}(\vec{r}, t) /|\phi|, \quad|\phi|^{2}=\phi^{a} \phi^{a}, \quad a=1,2 .
$$


The vortex current was given by [5]

$$
J_{v}^{\mu}=\frac{1}{2 \pi} \epsilon^{\mu \nu \lambda} \partial_{\nu} \bar{n} \frac{\partial_{\lambda}}{i} n
$$

By making use of the unit vector field $n^{a}$ the vortex current can be expressed as

$$
J_{v}^{\mu}=\frac{1}{2 \pi} \epsilon^{\mu \nu \lambda} \epsilon_{a b} \partial_{\nu} n^{a} \partial_{\lambda} n^{b}, \quad a, b=1,2 .
$$

It is clear that the vortex current is identically conserved, i.e.

$$
\partial_{\mu} j_{v}^{\mu}=0
$$

By making use of the $\phi$-mapping topological current theory, this vortex current can be rewritten in a compact form [6,7],

$$
j_{v}^{\mu}=D^{\mu}\left(\frac{\phi}{x}\right) \delta(\vec{\phi})
$$

where $D^{\mu}\left(\frac{\phi}{x}\right)$ is the vector Jacobians of $\phi(x)$ :

$$
D^{\mu}\left(\frac{\phi}{x}\right)=\frac{1}{2} \varepsilon^{\mu \nu \lambda} \varepsilon_{a b} \partial_{\nu} \phi^{a} \partial_{\lambda} \phi^{b}
$$

From the Eq. (3), we can see that vortex current $j_{v}^{\mu}$ does not vanish only at the zero points of $\phi$, i.e.

$$
\phi^{1}\left(x^{1}, x^{2}, t\right)=0, \quad \phi^{2}\left(x^{1}, x^{2}, t\right)=0 .
$$

The solutions of Eqs. (5) can be generally expressed as

$$
x^{1}=x_{l}^{1}(t), \quad x^{2}=x_{l}^{2}(t), \quad l=1,2, . ., N
$$

which represent $N$ zero points $\vec{z}_{l}(t)(l=1,2, \ldots, N)$ or worldlines of $N$ vortices in space-time. The location of $l$ th vortex is determined by the $l$ th zero point $\vec{z}_{l}(t)$.

According to the $\phi$-mapping topological current theory [6.7], one can prove that

$$
\delta^{2}(\vec{\psi})=\sum_{l=1}^{N} \frac{\beta_{l}}{\left|D\left(\frac{\phi}{x}\right)\right|_{\vec{z}_{l}}} \delta^{2}\left(\vec{r}-\vec{z}_{l}\right),
$$


where the positive integer $\beta_{l}$ is called the Hopf index of map $x->\phi$. The meaning of $\beta_{l}$ is that when the point $\vec{r}$ covers the neighborhood of the zero $\vec{z}_{l}$ once, the vector field $\vec{\phi}$ covers the corresponding region $\beta_{l}$ times. With the definition of vector Jacobians (4), we can obtain the general velocity of the $l$ th vortex

$$
v^{\mu}=\frac{d x_{l}^{\mu}}{d t}=\left.\frac{D^{\mu}(\phi / x)}{D(\phi / x)}\right|_{\vec{z}_{l}}, \quad v^{0}=1
$$

Then the vortex three-current $j_{v}^{\mu}$ can be written as the form of the current and the density of the system of $N$ classical point particles with topological charge $W_{l}=\beta_{l} \eta_{l}$ moving in the $(2+1)$-dimensional space-time

$$
\begin{aligned}
& \vec{j}=\sum_{l=1}^{N} W_{l} \vec{v}_{l} \delta^{2}\left(\vec{z}-\vec{z}_{l}(t)\right), \\
& \rho=j^{0}=\sum_{l=1}^{N} W_{l} \delta^{2}\left(\vec{z}-\vec{z}_{l}(t)\right),
\end{aligned}
$$

where $\eta_{l}=\operatorname{sgn}\left(\left.D(\phi / x)\right|_{\vec{z}_{l}}\right)= \pm 1$ is the Brouwer degree [7, 8]. It is clear to see that Eq. (9) shows the movement of the vortices in space-time.

\section{THE GENERATION AND ANNIHILATION OF VORTICES}

As being discussed before, the zeros of the condensate wave function $\psi$ play an important role in studying the vortices in the GL theory of the Fractional Quantum Hall Effect. Now, we begin studying the properties of the zero points (locations of vortices), in other words, the properties of the solutions of Eqs. (5). As we know before, if the Jacobian

$$
D\left(\frac{\phi}{x}\right)=\frac{\partial\left(\phi^{1}, \phi^{2}\right)}{\partial\left(x^{1}, x^{2}\right)} \neq 0,
$$

we will have the isolated solutions (6) of Eqs. (5). However, when the condition (10) fails, the usual implicit function theorem is of no use. The above results (6) will charge in some

way and will lead to the branch process. We denote one of the zero points as $\left(t^{*}, \vec{z}_{l}\right)$. If the Jacobian

$$
\left.D^{1}\left(\frac{\phi}{x}\right)\right|_{\left(t^{*}, \vec{z}_{l}\right)} \neq 0,
$$


we can use the Jacobian $D^{1}\left(\frac{\phi}{x}\right)$ instead of $D\left(\frac{\phi}{x}\right)$ for the purpose of using the implicit function theorem. Then we have an unique solution of Eqs. (5) in the neighborhood of the points $\left(t^{*}, \vec{z}_{l}\right)$

$$
t=t\left(x^{1}\right), \quad x^{2}=x^{2}\left(x^{1}\right),
$$

with $t^{*}=t\left(z_{l}^{1}\right)$. And we call the critical points $\left(t^{*}, \vec{z}_{l}\right)$ the limit points. In the present case, it is easy to know that

$$
\left.\frac{d x^{1}}{d t}\right|_{\left(t^{*}, \vec{z}_{l}\right)}=\frac{\left.D^{1}(\phi / x)\right|_{\left(t^{*}, \vec{z}_{l}\right)}}{\left.D(\phi / x)\right|_{\left(t^{*}, \vec{z}_{l}\right)}}=\infty
$$

i.e.

$$
\left.\frac{d t}{d x^{1}}\right|_{\left(t^{*}, \vec{z}_{l}\right)}=0
$$

The Taylor expansion of the solution of Eq. (12) at the limit point $\left(t^{*}, \vec{z}_{l}\right)$ is [6]

$$
t-t^{*}=\left.\frac{1}{2} \frac{d^{2} t}{\left(d x^{1}\right)^{2}}\right|_{\left(t^{*}, \vec{z}_{l}\right)}\left(x^{1}-z_{l}^{1}\right)^{2}
$$

which is a parabola in the $x^{1}-t$ plane. From Eq. (14), we can obtain two solutions $x_{1}^{1}(t)$ and $x_{2}^{1}(t)$, which give two branch solutions (worldlines of vortices) of Eqs. (5). If $\left.\frac{d^{2} t}{\left(d x^{1}\right)^{2}}\right|_{\left(t^{*}, \vec{z}_{l}\right)}>0$, we have the branch solutions for $t>t^{*}$, otherwise, we have the branch solutions for $t<t^{*}$. These two cases are related to the origin and annihilation of vortices.

From Eq. (13), we obtain an important result that the velocity of vortices is infinite when they are annihilating or generating, which is gained only form the topology of the condensate wave function.

Since the topological charge of vortices is identically conserved (2), the topological charges of these two vortices must be opposite at the limit point, i.e.,

$$
\beta_{l_{1}} \eta_{l_{1}}=-\beta_{l_{2}} \eta_{l_{2}}
$$

which shows that $\beta_{l_{1}}=\beta_{l_{2}}$ and $\eta_{l_{1}}=-\eta_{l_{2}}$. 


\section{BIFURCATION OF VORTEX THREE-CURRENT}

For a limit point, it also requires $\left.D^{1}(\phi / x)\right|_{\left(t^{*}, \vec{z}_{l}\right)} \neq 0$. As to a bifurcation point, it must satisfy a more complex condition at the bifurcation point $\left(t^{*}, \vec{z}_{l}\right)$ :

$$
\left\{\begin{array}{c}
\left.D(\phi / x)\right|_{\left(t^{*}, \vec{z}_{l}\right)}=0 \\
\left.D^{1}(\phi / x)\right|_{\left(t^{*}, \vec{z}_{l}\right)}=0
\end{array}\right.
$$

which will lead to an important fact that the function relationship between $t$ and $x^{1}$ is not unique in the neighborhood of the bifurcation point $\left(t^{*}, \vec{z}_{l}\right)$. It is easy to see from equation

$$
\left.\frac{d x^{1}}{d t}\right|_{\left(t^{*}, \vec{z}_{l}\right)}=\frac{\left.D^{1}(\phi / x)\right|_{\left(t^{*}, \vec{z}_{l}\right)}}{\left.D(\phi / x)\right|_{\left(t^{*}, \vec{z}_{l}\right)}}
$$

which under the restrain (16) directly shows that the direction of the integral curve of Eq. (17) is indefinite, i.e., the velocity field of vortices is indefinite at the point $\left(t^{*}, \vec{z}_{l}\right)$. This is why the very point $\left(t^{*}, \vec{z}_{l}\right)$ is called a bifurcation point of the condensate wave function.

Next, we will find a simple way to search for the different directions of all branch curves (or velocity field of vortex) at the bifurcation point. Assume that the bifurcation point $\left(t^{*}, \vec{z}_{l}\right)$ has been found from Eqs. (5) and (16). The Taylor expansion of the solution of Eqs. (5) in the neighborhood of the bifurcation point $\left(t^{*}, \vec{z}_{l}\right)$ can be expressed as [6]

$$
A\left(x^{1}-z_{1}^{1}\right)^{2}+2 B\left(x^{1}-z_{1}^{1}\right)\left(t-t^{*}\right)+C\left(t-t^{*}\right)^{2}=0
$$

which leads to

$$
A\left(\frac{d x^{1}}{d t}\right)^{2}+2 B \frac{d x^{1}}{d t}+C=0
$$

and

$$
C\left(\frac{d t}{d x^{1}}\right)^{2}+2 B \frac{d t}{d x^{1}}+A=0
$$

where $A, B$ and $C$ are three parameters. The solutions of Eq. (19) or Eq. (20) give different directions of the branch curves (worldlines of vortices) at the bifurcation point.

The remainder component $d x^{2} / d t$ can be given by 


$$
\frac{d x^{2}}{d t}=f_{1}^{2} \frac{d x^{1}}{d t}+f_{t}^{2}
$$

where partial derivative coefficients $f_{1}^{2}$ and $f_{t}^{2}$ have been calculated [6]. From these relations we find that the values of $d x^{2} / d t$ at the bifurcation point $\left(t^{*}, \vec{z}_{l}\right)$ are also possible different because (18) may give different values of $d x^{1} / d t$. The above solutions reveal the evolution of vortices. Besides the encountering of the vortices, i.e., two vortices encounter and then depart at the bifurcation point along different branch point, it may split into several vortices along different branch curves. On the contrary, several vortices can merge into one vortex at the bifurcation point. The identical conversation of the topological charge shows the sum of the topological charge of final vortices must be equal to that of the initial vortices at the bifurcation point, i.e.,

$$
\sum_{f} \beta_{j_{f}} \eta_{j_{f}}=\sum_{i} \beta_{j_{i}} \eta_{j_{i}}
$$

for fixed $j$. Furthermore, from above studies we see that the generation, annihilation and bifurcation of vortices are not gradual charges, but start at a critical value of arguments, i.e. a sudden charge.

\section{SKYRMIONS IN FQH EFFECT}

The $\nu=\frac{1}{m}$ FQH effect admits a Landau-Ginzburg description in terms of a complex

doublet of bosonic field $\Psi=\left(\begin{array}{c}\psi_{1} \\ \psi_{2}\end{array}\right)$ and a statistical Chern-Simons gauge field when the FQH effect system is depolarized [4,9,10]. We separate the magnitude and $S U(2)$ bases of $\Psi_{\sigma}: \Psi_{\sigma} \rightarrow \rho z_{\sigma}$ with $\overline{z_{\sigma}} z_{\sigma}=1$. The spin direction corresponding to $z_{\sigma}$ can be given by

$$
\Omega^{a}=\overline{z_{\alpha}} \sigma_{\alpha_{\beta}}^{a} z_{\beta}, \quad a=1,2,3
$$

where $\sigma^{a}$ are the Pauli matrices. The skyrmion three-current is [5]

$$
j_{s}^{\mu}=\frac{1}{4 \pi} \epsilon^{\mu \nu \lambda} \epsilon_{a b c} \Omega^{a} \partial_{\nu} \Omega^{b} \partial_{\lambda} \Omega^{c}, \quad \mu, \nu, \lambda=1,2,3 .
$$


It is well-known that this current can be expressed as [11]

$$
j_{s}^{\mu}=\frac{1}{4 \pi} \epsilon^{\mu \nu \lambda}\left(\partial_{\nu} A_{\lambda}-\partial_{\lambda} A_{\nu}\right)
$$

where $A_{\mu}=\frac{i}{2}\left(z_{\alpha}^{*} \partial_{\mu} z_{\alpha}-z_{\alpha} \partial_{\mu} z_{\alpha}^{*}\right)$, is essentially the Berry connection [12 with

$$
D_{\mu} \zeta=\partial_{\mu} \zeta-i A_{\mu} \zeta
$$

in which $\zeta=\zeta^{1}+i \zeta^{2}$ is a complex scalar field defined by the connection $A_{\mu}$. As one shown in [13], the $U(1)$ connection can be decomposed by the complex scalar field $\zeta$ as

$$
A_{\mu}=\epsilon^{a b} \partial_{\mu} m^{a} m^{b}+\partial_{\mu} \theta, \quad a=1,2
$$

in which $\theta$ is only a phase factor and $m^{a}=\zeta^{a} / \sqrt{\zeta^{b} \zeta^{b}}$ are the unit vector. With this connection the current (21) can be obtained

$$
j_{s}^{\mu}=\frac{1}{2 \pi} \epsilon^{\mu \nu \lambda} \epsilon_{a b} \partial_{\nu} m^{a} \partial_{\lambda} m^{b}
$$

Obviously, the current (22) is conserved. Following the $\phi$-mapping theory, it can be rigorously proved that

$$
j_{s}^{\mu}=\delta^{2}(\vec{\zeta}) D^{\mu}\left(\frac{\zeta}{x}\right)
$$

which is just similar to the vortex current $j_{v}^{\mu}$. But we have to point that the vector field $\zeta(x)$ in the skyrmions current is different from the spinless wave function $\phi(x)$ in the vortex current.

When the vector field $\vec{\zeta}$ possesses $l$ zeros, denoted as $z_{i}(i=1,2, \ldots, l)$, the skyrmions current can also be expressed by the winding number $W_{i}=\beta_{i} \eta_{i}$

$$
j_{s}^{\mu}=\left.\sum_{i=1}^{l} W_{i} \delta^{2}\left(\vec{x}-\vec{z}_{i}\right) \frac{d x^{\mu}}{d t}\right|_{z_{i}},
$$

which gives the density of skyrmions charge

$$
\rho=\sum_{i=1}^{l} W_{i} \delta^{2}\left(\vec{x}-\vec{z}_{i}\right) .
$$


Furthermore, the total charge of the system can be rewritten as

$$
Q=\int \rho(x) d^{2} x=\sum_{i=1}^{l} W_{i} .
$$

It is obvious that there exist $l$ isolated skyrmions of which the $i$ th skyrmion possesses charge $W_{i}$. And the skyrmion corresponds to $\eta_{i}=+1$, while the anti-skyrmion corresponds to $\eta_{i}=-1$.

\section{CONCLUSION}

First, in terms of the spinless wave function of the full polarized system, the inner topological structure of the vortex excitations is discussed with the $\phi$-mapping topological current theory. It is shown that the vortices in the FQH system are generated from $\phi=0$ and their topological charges are quantized under the condition $D(\phi / x) \neq 0$, i.e. the vortices emerge in a "natural way" as a solution of a topological current theory, instead of being put "by hand", as usually done in more phenomenological theories. One also shows that the charge of the vortex is determined by Hopf indices and Brouwer degrees. Second, we have studied the evolution of the vortices and concluded that there exist crucial cases of branch processes in the evolution of the vortices when $D(\phi / x)=0$, i.e., $\eta_{l}$ is indefinite. This means that the vortices are generated or annihilated at the limit points and are encountered, split, or merged at the bifurcation points of the wave function of the full polarized system, which shows that the vortices system is unstable at the branch points. Meantime, these conditions give simple rules to consider the nonlinear behavior in all sort of similar system. Third, with the skyrmions three-current given in [5] we obtain the inner structure of skyrmion excitations by making use of the decomposition of gauge potential theory. Finally, we would like to point that all the results in this paper have been obtained only from the viewpoint of topology without using any particular hypothesis. They will help those experts to find a branch point and the concrete branch process in the neighborhood of it, and give a deep insight into fractional quantum Hall system. 


\section{ACKNOWLEDGMENT}

This work was supported by the National Natural Science Foundation of China and the Doctoral Foundation of the People's Republic of China. 


\section{REFERENCES}

[1] D. C. Tsui, H. L. Stormer and A. C. Gossard, Phys. Rev. Lett. 48 (1982) 1559;

[2] R. B. Laughlin, Phys. Rev. Lett. 50 (1983) 1395;

[3] F. D. M. Haldane, Phys. Rev. Lett. 51 (1983) 605;

[4] S. L. Sondhi, A. Karlshede, S. A. Kivelson and E. H. Rezayi, Phys. Rev. B 47 (1993) 16419

[5] D. H. Lee and C. L. Kane, Phys. Rev. Lett. 64 (1990) 1313;

[6] Y. S. Duan, S. Li and G. H. Yang, Nucl. Phys. B514 (1998) 705;

[7] Y. S. Duan, H. Zhang and S. Li, Phys. Rev. B 58 (1998) 125;

[8] H. Hopf, Math. Ann. 96 (1929) 209;

[9] S. C. Zhang, H. Hansson and S. Kivelson, Phys. Rev. Lett. 62 (1989) 82; ibid. 62 (1989) 980; D. H. Lee and S. C. Zhang, Phy. Rev. Lett. 66 (1991) 1220; S. C. Zhang, Int. J. Mod. Phys. B6 (1992) 25;

[10] Z. F. Ezawa, Phys. Rev. B 55 (1997) 7771; ibid. Phys. Lett. A229 (1997) 392;

[11] E. Fradkin, Field Theories of Condensed Matter Systems (Addison-Wesley, New York, 1991).

[12] M. Stone, Phys. Rev. B 53 (1996) 16573;

[13] Y. S. Duan, in Proceedings of the Symposium on Yang-Mills Gauge Theories, Beijing, 1984; Y. S. Duan, G. H. Yang and Y. Jiang, Gen. Relativ. Gravit. 29 (1997) 715. 